\title{
Reliability of a Test Battery Designed for Quickly and Safely Assessing Diverse Indices of Neuromuscular Function
}

Barry A. Spiering ${ }^{1,2}$, Stuart M.C. Lee ${ }^{1}$, Ajitkumar P. Mulavara ${ }^{3}$, Jason R. Bentley ${ }^{1}$, Roxanne E. Buxton ${ }^{4}$, Emily L. Lawrence ${ }^{1}$, Joseph Sinka ${ }^{1}$, Mark E. Guilliams ${ }^{1}$, Lori L. Ploutz-Snyder ${ }^{3}$, Jacob J. Bloomberg ${ }^{5}$

${ }^{1}$ Wyle Integrated Science and Engineering Group, Houston, TX; ${ }^{2}$ California State University, Fullerton, CA; ${ }^{3}$ Universities Space Research Association, Houston, TX; ${ }^{4}$ University of Houston, Houston, TX; ${ }^{5}$ National Aeronautics and Space Administration Lyndon B. Johnson Space Center, Houston, TX 


\section{Introduction}

- Spaceflight affects nearly every physiological system

Spaceflight-induced alterations in physiological function translate to decrements in functional performance 


\section{Introduction}

- Challenge:

- How do we develop countermeasures to offset the plethora of physiological decrements?

- Solution:

- Identify the physiological factors most critical for functional outcomes

- Develop countermeasures targeted toward the most critical factors 


\section{Introduction}

Functional Performance

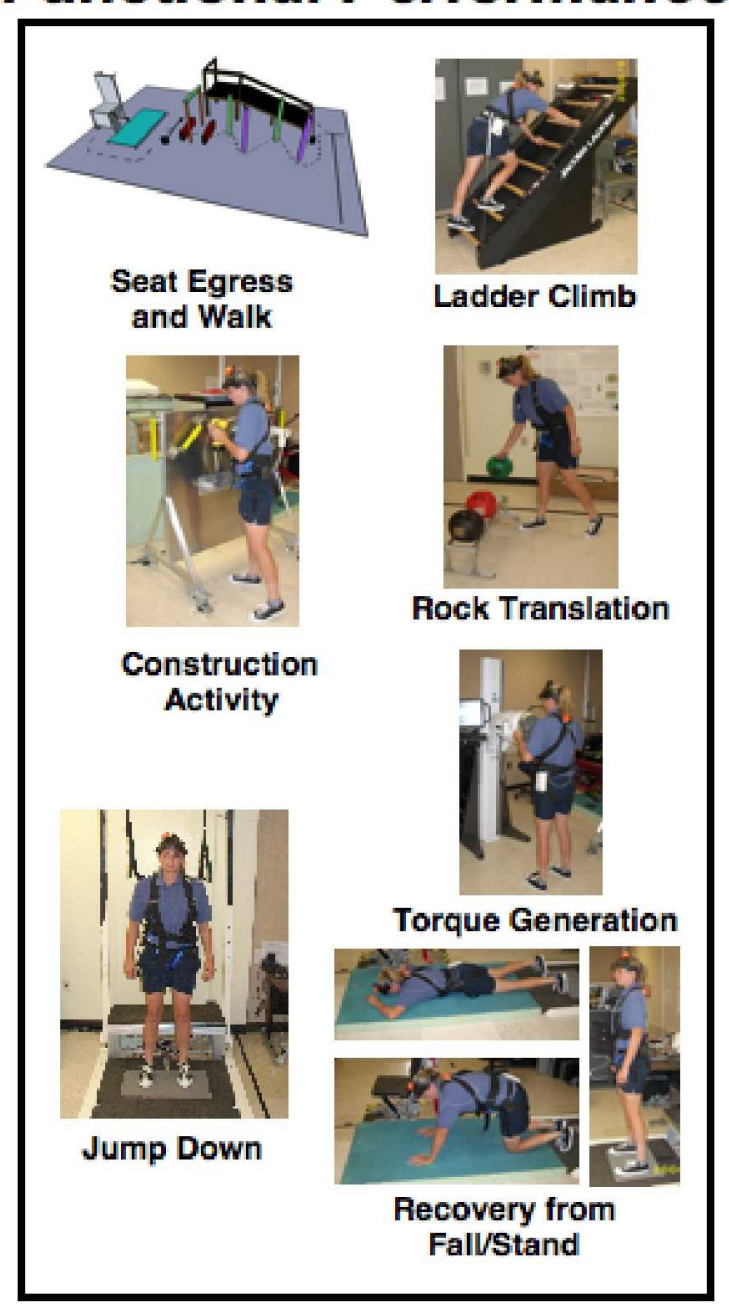

\section{Physiological Measures}

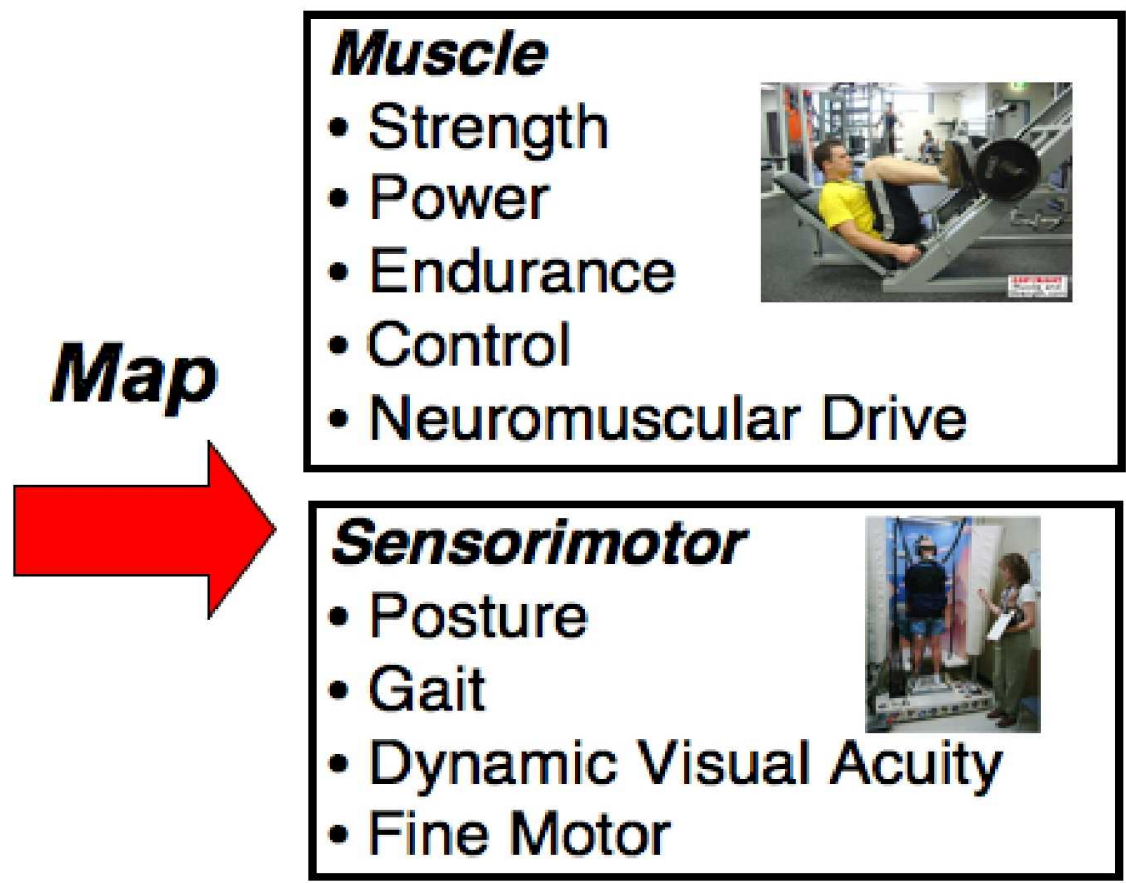

Cardiovascular

- Plasma Volume 


\section{What "Neuromuscular Performance Variables" Do We Assess?}

- Reduced strength is a hallmark consequence of spaceflight

- Strength is strongly associated with functional performance (Visser et al. 2000)

- "Neural factors" (e.g., central activation) clearly contribute to unloadinginduced strength loss (Clark et al. 2006)

- Power is perhaps the strongest predictor of functional performance (Puthoff et al. 2008)

- Force steadiness might relate to functional performance (Seynnes et al. 2005; Manini et al. 2005) 


\section{Purpose}

- To develop a test battery for quickly and safely assessing diverse indices of neuromuscular performance

- Quickly:

- Battery of tests must be completed in $\sim 30 \mathrm{~min}$

- Safely:

- Increased susceptibility to muscle damage after spaceflight

- Impaired postural stability post-spaceflight

- Diverse indices:

- Strength

- Central activation

- Power

- Endurance

- Force steadiness 


\section{Methods}

- Subjects

- 10 healthy volunteers ( 5 women, 5 men)

- Age: $31 \pm 5$ y

- Height: $173 \pm 11 \mathrm{~cm}$

- Weight: $73 \pm 14 \mathrm{~kg}$

Procedures

- Completed a battery of neuromuscular performance tests on 3 occasions separated by at least $48 \mathrm{~h}$ 


\section{Knee Extension Tests}

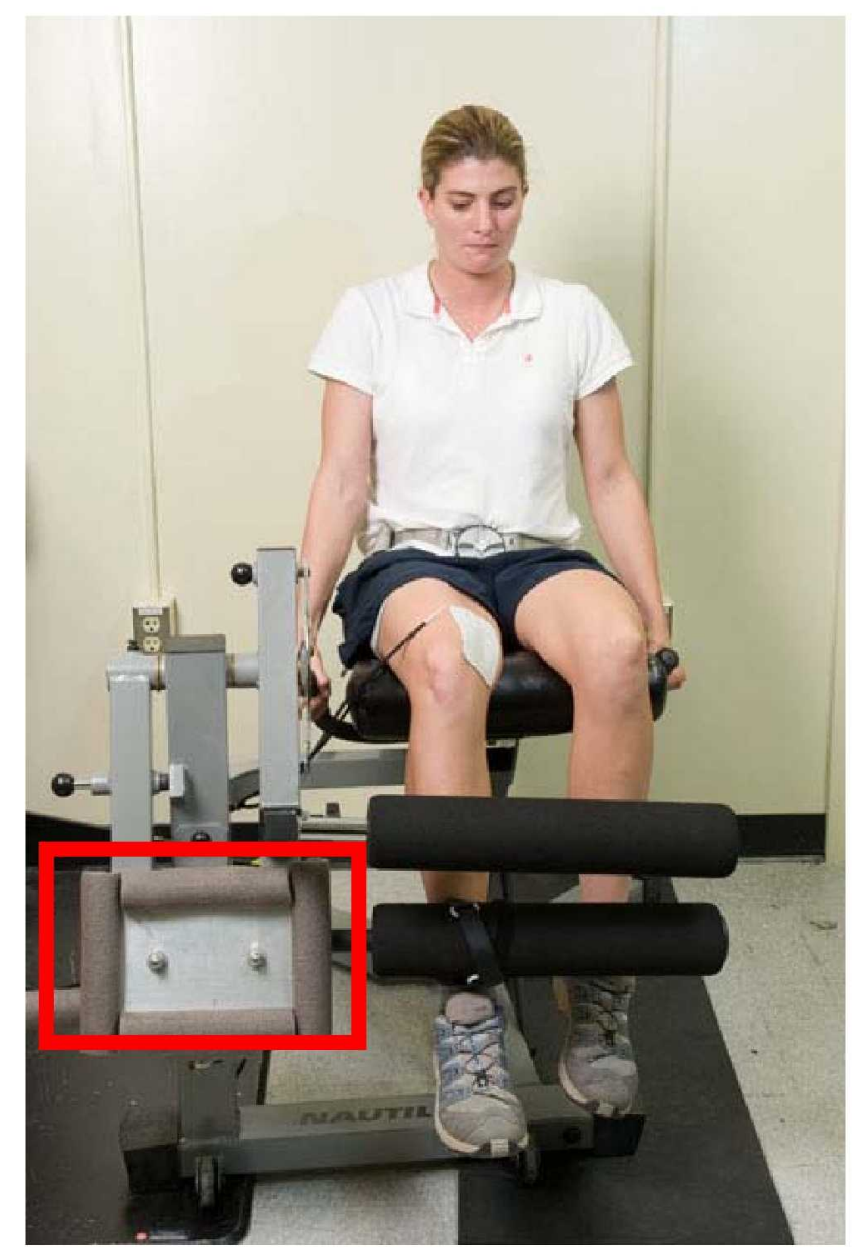

Test \#1: Interpolated Twitch Test

-Central Activation

\section{Test \#2: Force Steadiness Test}

-With and without Visual Feedback 


\section{Bench Press Tests}

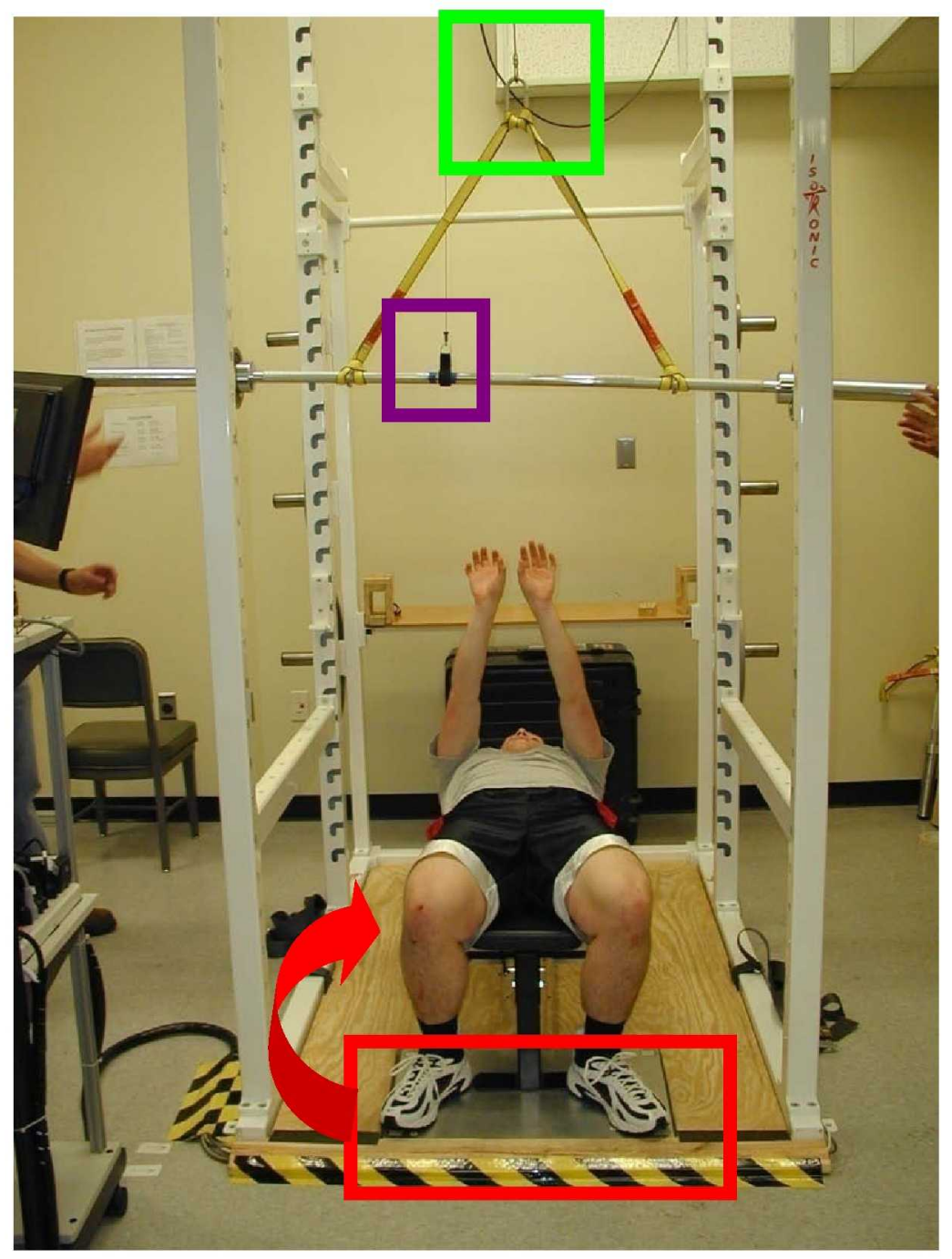

Test \#5: Maximal Isometric Force Test

Maximal Strength

-Rate of Force Development

Test \#6: Force Steadiness Test

-With and without Visual Feedback

Test \#7: Power Endurance Test

-Maximal Power

-Fatigue Index

-Total Work 


\section{Statistical Analyses}

- Reliability of each test was assessed via

- Standard error of the measurement (SEM)

- SEM reported as percent of the mean

- Intraclass correlation coefficient (ICC)

- Hme required to set up and conduct each test is reported as mean \pm SD 


\section{Results: Knee Extension Tests}

\begin{tabular}{|l|l|l|l|}
\hline Test & Dependent Variable & SEM & ICC \\
\hline Interpolated Twitch & Central Activation Capacity (\%) & $3 \%$ & 0.87 \\
\hline Force Steadiness & $\begin{array}{l}\text { Force Steadiness with Visual } \\
\text { Feedback (CV) }\end{array}$ & $35 \%$ & 0.20 \\
\hline & $\begin{array}{l}\text { Force Steadiness without Visual } \\
\text { Feedback (CV) }\end{array}$ & $35 \%$ & 0.28 \\
\hline
\end{tabular}




\section{Results: Leg Press Tests}

\begin{tabular}{|c|c|c|c|}
\hline Test & Dependent Variable & SEM & ICC \\
\hline \multirow[t]{2}{*}{ Maximal Isometric Force } & Maximal Isometric Force (N) & $4 \%$ & 0.99 \\
\hline & $\begin{array}{l}\text { Rate of Force Development } \\
(\mathrm{N} / \mathrm{ms})\end{array}$ & $9 \%$ & 0.94 \\
\hline \multirow[t]{3}{*}{ Power Endurance } & Maximal Power (W) & $3 \%$ & 0.99 \\
\hline & Fatigue Index (\%) & $18 \%$ & 0.36 \\
\hline & Total Work (J) & $4 \%$ & 0.99 \\
\hline
\end{tabular}




\section{Results: Bench Press Tests}

\begin{tabular}{|c|c|c|c|}
\hline Test & Dependent Variable & SEM & ICC \\
\hline \multirow[t]{2}{*}{ Maximal Isometric Force } & Maximal Isometric Force (N) & $3 \%$ & 0.99 \\
\hline & $\begin{array}{l}\text { Rate of Force Development } \\
(\mathrm{N} / \mathrm{ms})\end{array}$ & $14 \%$ & 0.93 \\
\hline \multirow[t]{2}{*}{ Force Steadiness } & $\begin{array}{l}\text { Force Steadiness with Visual } \\
\text { Feedback }(\mathrm{CV})\end{array}$ & $20 \%$ & 0.60 \\
\hline & $\begin{array}{l}\text { Force Steadiness without Visual } \\
\text { Feedback (CV) }\end{array}$ & $33 \%$ & 0.26 \\
\hline \multirow[t]{3}{*}{ Power Endurance } & Maximal Power (W) & $9 \%$ & 0.97 \\
\hline & Fatigue Index (\%) & $16 \%$ & 0.62 \\
\hline & Total Work (J) & $4 \%$ & 0.99 \\
\hline
\end{tabular}




\section{Results: Time Requirements}

\begin{tabular}{|c|c|c|c|c|c|c|c|}
\hline \multirow{2}{*}{$\begin{array}{l}\text { Testing Device } \\
\text { Knee Extension }\end{array}$} & \multirow{2}{*}{$\begin{array}{l}\text { Test } \\
\text { ITT Current Optimization }\end{array}$} & \multicolumn{2}{|c|}{ Session 1} & \multicolumn{2}{|c|}{ Session 2} & \multicolumn{2}{|c|}{ Session 3} \\
\hline & & 11 & 4 & 9 & 3 & 9 & 2 \\
\hline & Interpolated Twitch & 7 & 2 & 6 & 2 & 7 & 3 \\
\hline & Force Steadiness & 4 & 2 & 5 & 2 & 5 & 2 \\
\hline \multirow[t]{2}{*}{ Leg Press } & Maximal Isometric Force & 8 & 4 & 6 & 1 & 7 & 2 \\
\hline & Power Endurance & 3 & 1 & 2 & 1 & 2 & 0 \\
\hline \multirow[t]{4}{*}{ Bench Press } & Maximal Isometric Force & 5 & 2 & 4 & 1 & 4 & 1 \\
\hline & Force Steadiness & 6 & 3 & 5 & 2 & 5 & 1 \\
\hline & Power Endurance & 2 & 1 & 2 & 1 & 3 & 1 \\
\hline & Total & 46 & 6 & 39 & 5 & 40 & 6 \\
\hline
\end{tabular}




\section{Conclusions}

- Purpose: To develop a test battery for quickly and safely assessing diverse indices of neuromuscular performance

- Quickly:

- Battery of tests can be completed in $\sim 30-40$ min

- Safely:

- No eccentric muscle actions or impact forces

- Tests present little challenge to postural stability

- Diverse indices:

- Strength: Excellent reliability $(I C C=0.99)$

- Central activation: Very good reliability $(I C C=0.87)$

- Power: Excellent reliability $(I C C=0.99)$

- Endurance: Total work has excellent reliability $(I C C=0.99)$

- Force steadiness: Poor reliability (ICC $=0.20-0.60)$ 


\section{Acknowledgments}

- This work was supported by the Exercise Physiology and Countermeasures Project of the National Aeronautics and Space Administration

We thank Brent Crowell, Kirk English, Jamie Guined, Mark Leach, Peggy Lynn, and Leah Stroud for invaluable assistance during data collection

I 


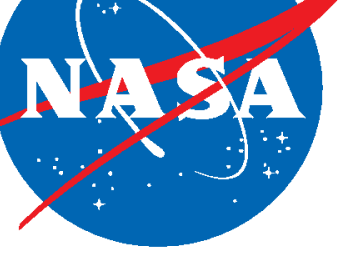

